[RAdiocarbon, Vol. 19, No. 1, 1977, P. 49-61]

\title{
MONACO RADIOCARBON MEASUREMENTS V
}

\section{J L RAPAIRE and G HUGUES}

Centre Scientifique De Monaco, Monaco

Most of the ${ }^{14} \mathrm{C}$ measurements reported here were made between October 74 and March 76. The usual procedures and methods of sample preparation are those described in R, 1964, v 6, p 194-196. The electronic apparatus was constructed by $\mathrm{J}$ Galliot from results of studies on background of proportional counters (Delibrias \& Rapaire, 1967) and was described briefly in R, 1966, v 8, p 286-291.

Age calculations are based on a contemporary value equal to 0.95 of the activity of NBS oxalic acid and conventional half-life for ${ }^{14} \mathrm{C}$ of 5568 years. Given errors are $\pm 1 \sigma$, and are calculated according to the method described in $\mathrm{R}, 1965, \mathrm{v} 7$, p 156-161. The proportional counter is filled with $\mathrm{CO}_{2}$ at a pressure of $740 \pm 1 \mathrm{~mm} \mathrm{Hg}$. The statistical error on the counting rate of background and NBS standard are equivalent to a counting time of $4000 \mathrm{~min}$. When $\mathrm{CO}_{2}$ of the sample is insufficient, inactive $\mathrm{CO}_{2}$ is added. No measurements of $\delta^{13} \mathrm{C}$ are made.

\section{ACK NOWLEDGMENTS}

We gratefully acknowledge the help of Y Thommeret in the maintenance of the chemical laboratory. We thank also the staff of the Mus Oceanograph, Monaco, the captain and the crew of $R / V$ WinnarettaSinger for their assistance in our work, and all the submitters who sent us their comments.

\section{SAMPLE DESCRIPTIONS}

I. SOIL SAMPLES

These measurements are used to determine the residence time of fulvic acids, soluble in an acid solution, humic acids, precipitated in an acid solution, and non-extractable fraction in alkaline reactives, or humin, in a soil assumed to be in equilibrium. The box model and the equations are given by Lobo (1974). Extraction technique uses $\mathrm{Na}_{4} \mathrm{P}_{2} \mathrm{O}_{7}$ at $\mathrm{pH} 10$ and sample weight is usually $1 \mathrm{~kg}$. Measurements are given in apparent ages.

\section{Pariacabo series}

The Savannah of Pariacabo $\left(5^{\circ} 10^{\prime} \mathrm{N}, 52^{\circ} 39^{\prime} \mathrm{W}\right)$, French Guyana, alt $+10 \mathrm{~m}, 3 \mathrm{~km}$ from sea. Topography is characteristic of an old offshore bar deposit, with undulations smoothed by erosion and warping. Progressive lateral change between hydromorphic podzols with humic hard pan (plinthaqultic tropaquod) can be observed at the summit of the landscape, and ferrallitic soils (dystropeptic haplorthox or plinthic ochraqult) towards the base (Turenne, 1975). Samples represent progressive individualization in the deep profile, of a humic accumulation level, and correspond each time to the maximum of deep organic matter accumulation observed. Only PAR 91 comes from the surface. Samples coll and 
subm 1973 by J F Turenne, Office Recherche Sci \& Tech Outre Mer (ORSTOM), Bondy, France.

MC-1020. PAR 91, humic acids

$\delta^{14} \mathrm{C}=+128 \pm 16 \%$ o

Level 1 to $13 \mathrm{~cm}$; carbon content $1.1 \%$; dilution factor 2 .
MC-1021. PAR 91, humin
$\delta^{14} \mathrm{C}=+\mathbf{2 1 7} \pm \mathbf{1 1} \%$
Level 1 to $13 \mathrm{~cm}$; carbon content $5.6 \%$.
MC-1022. PAR 91, fulvic acids
$\delta^{14} \mathrm{C}=+427 \pm 43 \%$

Level 1 to $13 \mathrm{~cm}$; carbon content $0.5 \%$; dilution factor 6 .

MC-1008. PAR 43, humic acids

$$
270 \pm 150
$$

Level 37 to $47 \mathrm{~cm}$; carbon content $0.6 \%$; dilution factor 4 .

MC-1009. PAR 43, humin AD 1380

$570 \pm 210$

Level 37 to $47 \mathrm{~cm}$; carbon content $3 \%$; dilution factor 1.3 .

MC-1010. PAR 43, fulvic acids

$1030 \pm 90$

Level 37 to $47 \mathrm{~cm}$; carbon content $2.2 \%$.

AD 920

MC-1017. PAR 54, humic acids

$\delta^{14} \mathbf{C}=+\mathbf{2 8} \pm \mathbf{5 6} \%$

Level 55 to $70 \mathrm{~cm}$; carbon content $0.6 \%$; dilution factor 8 .

MC-1018. PAR 54, humin

Level 55 to $70 \mathrm{~cm}$; carbon content $3.1 \%$.

MC-1019. PAR 54, fulvic acids

Level 55 to $70 \mathrm{~cm}$; carbon content $2.8 \%$.

MC-1014. PAR 85, humic acids

Level 74 to $82 \mathrm{~cm}$; carbon content $1.1 \%$; dilution factor 4 .

MC-1015. PAR 85, humin

Level 74 to $82 \mathrm{~cm}$; carbon content $2.5 \%$.

MC-1016. PAR 85, fulvic acids

Level 74 to $82 \mathrm{~cm}$; carbon content $7 \%$.

MC-1004. PAR 95, humic acids

Level 82 to $96 \mathrm{~cm}$; carbon content $1.7 \%$; dilution factor 2.

MC-1005. PAR 95, humin

Level 82 to $96 \mathrm{~cm}$; carbon content $2.3 \%$.
$2270 \pm 100$

320 вC

$2500 \pm 100$

550 BC

$5270 \pm 460$

3360 BC
$3560 \pm 90$

$1610 \mathrm{BC}$

$3300 \pm 100$

$1350 \mathrm{BC}$

$11,300 \pm 270$

9350 BC

$7250 \pm 100$

5300 BC 
MC-1006. PAR 95, fulvic acids

$7160 \pm 120$

Level 82 to $96 \mathrm{~cm}$; carbon content $12.5 \%$.

5210 BC

General Comment (JFT): apparent age determination of Bh horizons and those of different organic fractions composing them enable understanding of stages of podzolization. Two conclusions can be drawn: 1) for a mean age gradient increasing towards the summit, podzolization began at the summit of the soil chain and progressed to the base; 2) two processes can explain age gradient differing according to the humic fraction: a) insolubilization of young precursors producing humic acids; in the 1st stages of formation of Bh level. Modern age of these components: MC1017; MC-1008. b) maturation and slow bio-degradation that affect inequally all fractions. Humic acids appear to undergo, from the moment of their insolubilization in the profile, the weakest renovation. Their age represents, at the top, the beginning of their insolubilization and gets a lower limit of the start of podzolization, 11,000 yr вр.

Lateral movement of podzolization front corresponds to a progressive rise of water level appearing 1st at the center of the location and then invading all the landscape; this is in connection with the progressive warping of water courses and rise of the sea level, diminishing drainage flow in the savannahs.

\section{ARCHAEOLOGIC SAMPLES}

\section{A. Argentina, Tierra del Fuego}

\section{Lancha Packewaia series}

Six charcoal samples coll from a stratified shell midden on $\mathrm{N}$ shore of Beagle channel $\left(54^{\circ} 49^{\prime} \mathrm{S}, 68^{\circ} 10^{\prime} \mathrm{W}\right)$ Jan-Feb 1975 , by A E Sala, L A Orquera, E L Piana, A H Tapia, Fund Antropol Argentina, Zarnatu 1375, Buenos Aires, subm by E C Saxon, Dept Antropol, Univ Durham, England.

\section{MC-1062. Lancha Packewaia VI/13/B \\ $280 \pm 85$ \\ MC-1064. Lancha Packewaia III/10/B \\ AD 1670 \\ $280 \pm 85$}

Samples from uppermost midden levels of 2 trenches $30 \mathrm{~m}$ apart. Site is on fossil beach, at $+7 \mathrm{~m}, 18 \mathrm{~km} \mathrm{E}$ of Ushuaia.

\section{MC-1066. Lancha Packewaia III/14/C}

$410 \pm 75$

MC-1063. Lancha Packewaia VI/14/C

AD 1540

$455 \pm 85$

AD 1495

Samples from humus layers underlying uppermost midden levels. Tree-ring calibration places upper midden in 16th century and underlying soil in 15th century (Stuiver and Suess, 1966). 
MC-1065. Lancha Packewaia VI/18/D

$$
1080 \pm 100
$$

AD 870 "C".

Sample from middle midden layer, stratigraphically underlying Soil

MC-1068. Lancha Packewaia VI/21/E

$4215 \pm 305$ AD 2265

Sample from surface of fossil beach, underlying 3rd lowest shell midden. Too old, age may be considered maximum. Sample diluted with old $\mathrm{CO}_{2}$. Dilution factor: 4.

General Comments (ECS): 3 occupation periods recorded at this shell midden correspond well with those at Lauta, Isla Navarino, Chile, on opposite shore of Beagle Channel from Lancha Packewaia (OrtizTroncoso, 1971). Upper units at Lauta are exactly contemporaneous, suggesting gap in occupation between Ca AD 1100 and 1500 is real (Lauta 1: Gif-2728: $280 \pm$ 90; Lauta 1a: R-45-42/1: $1080 \pm 60$; Lauta 3: Gif-2729: $2780 \pm 110)$. The Ona occupation of the continental site, Fell's Cave, began during this interval (Fell's Cave V: I-5139: $685 \pm 90$ ) which corresponds to period of secondary climatic optimum in Britain. (LAO): 2 cultural phases for region, defined by Bird (1938), are not clearly distinguishable at Lancha Packewaia. Upper midden ("B") contains some glass, iron objects, and a European sherd. Although Beagle Channel was not discovered until 1829, the Yamana Indians were occasional visitors to other places and tribes, already in contact with Europeans in the 16th century. Industry of Upper Midden includes spear points resembling several continental Patagonian types. The distinctive spear point of the Middle and Lower Middens is bipointed and leaf-shaped, but rather thick. Single-shouldered bone harpoons were present in Upper Midden, while Lower Midden is distinguished by its cruciform-base harpoons, as reported from early littoral sites in neighboring Chile (Ortiz-Troncoso, 1973). Also present in this level are bifaces, coarse pebble tools, and bolas.

\section{Northern Tierra del Fuego series}

Three samples from grassland zone known to have been occupied by various Indian cultures, Gif-1034: $9590 \pm 210$, until European conquest ca AD 1895. Coll Jan 1975 and subm by E C Saxon.

\section{MC-1069. Cabeza de Leon}

$$
1100 \pm 95
$$

\section{AD 850}

Wood charcoal $\left(53^{\circ} 19^{\prime} \mathrm{S}, 68^{\circ} 35^{\prime} \mathrm{W}\right)$ from depth $30 \mathrm{~cm}$, within rock shelter. Assoc artifacts show fine pressure flaking.

\section{MC-1070. Tres Arroyos}

$$
135 \pm 85
$$

\section{AD 1815}

Wood charcoal $\left(53^{\circ} 22^{\prime} \mathrm{S}, 68^{\circ} 50^{\prime} \mathrm{W}\right)$ from depth $50 \mathrm{~cm}$, at rear of $\mathbf{N}$ facing rock shelter. Tres Arroyos, Chile, and Cabeza de Leon, Argentina are the only stratified inland sites known on Tierra del Fuego. 
MC-1071. Castillo

Modern

Charcoal flecks $\left(53^{\circ} 40^{\prime} \mathrm{S}, 68^{\circ} 28^{\prime} \mathrm{W}\right)$ from wind-eroded scarp under collapsed rock shelter. Area has several surface sites, but no other shelters.

\section{B. Brazil, Pernambuco}

All materials found at the following sites, except that of Derbi, are described by A F G Laroche (1975).

\section{Pedra do Caboclo series}

Charcoals from levels of archaeol site formed by numerous caves and rock shelters at alt 290m near Bom Jardim ( $7^{\circ} 47^{\prime} 59^{\prime \prime} \mathrm{S}, 35^{\circ} 35^{\prime} 35^{\prime \prime} \mathrm{W}$ ). Samples coll and subm 1974 by A F G Laroche, Col Estadual Pernambuco, Brazil.

MC-1003. Pedra do Caboclo, Rock Shelter 1

$8400 \pm 200$

Sq la, level $100 \mathrm{~cm}$.

\section{MC-1025a. Pedra do Caboclo, Rock Shelter 2}

Sq $1 \mathrm{~b}$, level 30 to $40 \mathrm{~cm}$. Comment: another sample of same origin was dated at : $145 \pm 85$; MC-1025b.

\section{MC-1040. Pedra do Caboclo, Rock Shelter 2}

Sq 16 , level 50 to $60 \mathrm{~cm}$.

MC-1024. Pedra do Caboclo, Rock Shelter 2

Sq $1 \mathrm{~b}$, level 80 to $90 \mathrm{~cm}$.

MC-1051. Pedra do Caboclo, Rock Shelter 2

Sq lb, level 110 to $130 \mathrm{~cm}$.

$$
2900 \pm 95
$$

950 вC

$$
\begin{array}{r}
300 \\
\text { AD } 1650
\end{array}
$$

MC-1023a. Pedra do Caboclo, Rock Shelter $2 \quad 1500$ вC

Sq 16, level 150 to $180 \mathrm{~cm}$; dilution factor: 9. Comment: rest of sample, $10 \mathrm{~g}$ charcoal earth, was dated $250 \pm \underset{950}{\operatorname{Modern}}$, MC-1023b; dilution factor: 9. Sample contaminated with debris of rootlets.

MC-1041. Pedra do Caboclo, Cave 2

$380 \pm 70$

Sq 8 , level 0 to $10 \mathrm{~cm}$.

MC-1034. Pedra do Caboclo, Cave 2

Sq 4 , level 140 to $170 \mathrm{~cm}$.

MC-1039. Pedra do Caboclo, Rock Shelter 3 AD 1580

Sq 6 , level 40 to $60 \mathrm{~cm}$.

$1775 \pm 100$ AD 180

$2620 \pm 90$

670 вC 
MC-1036. Pedra do Caboclo, Rock Shelter 3

$4515 \pm 115$

Sq 6 , level 60 to $80 \mathrm{~cm}$.

MC-1037. Pedra do Caboclo, Rock Shelter 3

$6085 \pm 120$

Sq 6 , level 80 to $100 \mathrm{~cm}$.

\section{MC-1038. Pedra do Caboclo, Rock Shelter $3 \quad 4275$ BC}

Sq 6, level 100 to $120 \mathrm{~cm}$.

General Comment (AFGL): lithic material from level dated by MC-1003 is similar to that found by V Calderon (pers commun) in 1967 at "Grotta do Padre" $7580 \pm 140$, Si-544. MC-1003 belongs to "Tradiçao Itaparica, subtradiçao Bom Jardim". Samples MC-1024, 1034, 1041 represent "Tradiçao ceramica da Pedra do Caboclo". The others correspond to cultural phases not yet identified. Site described by A F G Laroche (1970).

\section{Angico series}

Charcoals from levels of archaeol site formed by 1 cave and 2 grottoes at alt 316 to $350 \mathrm{~m}$ near Bom Jardim $\left(7^{\circ} 48^{\prime} 25^{\prime \prime} \mathrm{S}\right.$, $\left.35^{\circ} 32^{\prime} 20^{\prime \prime} \mathrm{W}\right)$. Samples coll and subm in 1974 by AFG Laroche.

MC-1031. Angico Funeral Cave 2

$1515 \pm 80$

Sq C, level $40 \mathrm{~cm}$. AD 435

MC-1035. Angico Funeral Cave 2 Sq E, level $35 \mathrm{~cm}$.

$$
300 \pm 85
$$
AD 1650

MC-1002. Angico, Grotte S $440 \pm 50$ Sq 3 , level $70 \mathrm{~cm}$.

\section{MC-1011. Angico, Grotte S}

Sq 3, level $125 \mathrm{~cm}$.

$$
\mathbf{5 4 0} \pm \mathbf{7 0}
$$

\section{MC-1007. Angico, Grotte N}

$4650 \pm 150$

Sq A, level $110 \mathrm{~cm}$.

2700 BC

General Comment (AFGL): MC-1007 related to culture "fase preceramica Paquevira", the other samples to "tradiçao ceramica da Pedra do Caboclo". MC-1002 and 1031 are charcoal from funeral urns. Site described by AFG Laroche (1969, 1973, 1976).

\section{Cercado series}

Charcoal from rock shelter on right bank of Orobo $\mathrm{R}\left(7^{\circ} 43^{\prime} 20^{\prime \prime} \mathrm{S}\right.$, $\left.35^{\circ} 31^{\prime} 10^{\prime \prime} \mathrm{W}\right)$ at alt $270 \mathrm{~m}$ near "Fazenda Paquevira". Samples coll and subm 1973 by AFG Laroche. 
MC-1030. Cercado

$1295 \pm 95$

Sq 2, level $65 \mathrm{~cm}$.

AD 655

MC-1033a. Cercado

$1010 \pm 85$

Sq A, level $30 \mathrm{~cm}$.

AD 940

MC-1033b. Cercado

Modern

Sq C, level $30 \mathrm{~cm}$.

General Comment (AFGL): site corresponds to "tradiçao ceramica da Pedra do Caboclo".

\section{Coati}

Charcoal from rock shelter $\left(7^{\circ} 47^{\prime} 59^{\prime \prime} \mathrm{S}\right.$, $\left.35^{\circ} 35^{\prime} 23^{\prime \prime} \mathrm{W}\right)$ near Bom Jardim on bank of stream at alt $300 \mathrm{~m}$; coll and subm 1974 by AFG Laroche.

\section{MC-1032. Coati}

$$
295 \pm 85
$$

Sq 2, level $30 \mathrm{~cm}$.

General Comment (AFGL): level contains ceramic, lithic objects, ornaments, human remains represent end of "tradiçao ceramica da Pedra do Caboclo".

\section{MC-1012. Nunes}

$$
195 \pm 75
$$

\section{AD 1755}

Charcoal from cave top of rocky escarpment, Sq N, level $150 \mathrm{~cm}\left(7^{\circ}\right.$ $\left.43^{\prime} 20^{\prime \prime} \mathrm{S}, 35^{\circ} 30^{\prime} 59^{\prime \prime} \mathrm{W}\right)$ near Paquevira, at alt 280m; coll 1974 by A Soares and subm by AFG Laroche. Comment (AFGL): very coarse lithic culture. Date is too young.

\section{MC-1047. Camara}

$195 \pm 75$

Charcoal from rock shelter, Sq 1, level 30 to $40 \mathrm{~cm}\left(7^{\circ} 44^{\prime} 30^{\prime \prime} \mathrm{S}\right.$, $35^{\circ} 33^{\prime} 11^{\prime \prime} \mathrm{W}$ )near Bom Jardim, coll and subm 1974 by AFG Laroche. Comment (AFGL): recent indigenous occupation.

\section{Derbi series}

Charcoals from levels of open-air archaeol site $\left(7^{\circ} 47^{\prime} 35^{\prime \prime} \mathrm{S}, 35^{\circ} 35^{\prime}\right.$ $23^{\prime \prime} \mathrm{W}$ ) at alt $360 \mathrm{~m}$ on bank of Tracunhaém R, outside urban perimeter of town Bom Jardim. Samples coll and subm 1975 by AFG Laroche.

\section{MC-1073. Derbi}

Sq 1 , level 10 to $20 \mathrm{~cm}$.

MC-1074. Derbi

Sq 3, level 0 to $10 \mathrm{~cm}$.
$1470 \pm 270$ AD 480

$$
1510 \pm 150
$$

AD 440 
MC-1075. Derbi

Sq 4, level 0 to $10 \mathrm{~cm}$.

MC-1076. Derbi

Sq 4, level 10 to $20 \mathrm{~cm}$; dilution factor: 3.8 .

MC-1077. Derbi

Sq 5, level 0 to $10 \mathrm{~cm}$.

MC-1079. Derbi

Sq 5, level 20 to $30 \mathrm{~cm}$.

\section{MC-1080. Derbi}

$730 \pm 115$

AD 1220

$620 \pm 100$ AD 1330

Modern

$$
1050 \pm 120
$$

AD 900

$$
\begin{array}{r}
1100 \\
\text { AD } 850
\end{array}
$$

cm. Comment (AFGL): samples are rustic implements of quartz made with quadrangular uniface nuclei. Industry was named "fase Itagiba". MC-1077 and - 1079 are too young.

\section{Chã do Caboclo series}

Charcoals from different levels of open-air archaeol site $\left(7^{\circ} 44^{\prime} 30^{\prime \prime}\right.$ $\mathrm{S}, 35^{\circ} 33^{\prime} 10^{\prime \prime} \mathrm{W}$ ), on a plateau at alt $420 \mathrm{~m}$, Espera dist near Bom Jardim. Topography of site was levelled by erosion. Four strata, depths of which vary according to loc from 0 to $80 \mathrm{~cm}$ in relation to actual level of soil, have been found. Samples coll and subm 1974 and 1975 by AFG Laroche.

MC-1053. Chã do Caboclo, Stratum A1

Sq 39 , level 10 to $20 \mathrm{~cm}$.

MC-1054. Chã do Caboclo, Stratum Al

Sq 39 , level 30 to $40 \mathrm{~cm}$.

MC-1057. Chã do Caboclo, Stratum Al

Sq 45 , level 0 to $10 \mathrm{~cm}$.

MC-1058. Chã do Caboclo, Stratum A1

Sq 45 , level $50 \mathrm{~cm}$.
$370 \pm 95$
AD 1580

$$
270 \pm 85
$$

AD 1680

AD 1760

$190 \pm 90$
$295 \pm 85$
AD 1655

$295 \pm 75$

\section{MC-1088. Chã do Caboclo, Stratum A1}

AD 1655

Sq 53, level 10 to $20 \mathrm{~cm}$. Comment (AFGL): samples are related to a neo-Brazilian ceramic culture with "Tupiguarani" and "Cariri" influences. MC-1053 and -1054 from charcoal silo. Age of MC-1058 is too young since it does not correspond to material found at that level, which is most probably due to rearrangement of soil around Sq 45. 
MC-1082. Chã do Caboclo, Stratum A2

$360 \pm 135$

Sq 51 , level 10 to $20 \mathrm{~cm}$.

AD 1590

MC-1083. Chã do Caboclo, Stratum A2

$330 \pm 110$ AD 1620

Sq 51, level 30 to $40 \mathrm{~cm}$. Comment (AFGL): stratum contains red and white fragments of tupiguarani ceramic "fase cacimba-subtradiçao pintada".

MC-1052. Chã do Caboclo, Stratum A3

$945 \pm 85$

Sq 36 , level 0 to $10 \mathrm{~cm}$.

AD 1005

MC-1086. Chã do Caboclo, Stratum A3

$1995 \pm 85$

Sq 52 , level 10 to $20 \mathrm{~cm}$.

$45 \mathrm{BC}$

MC-1026. Chã do Caboclo, Stratum A3

$2025 \pm 95$

$75 \mathrm{BC}$

Sq 3, level 10 to $20 \mathrm{~cm}$. Comment (AFGL): stratum corresponds to maximum expansion of "tradiçao ceramica da Pedra do Caboclo" and to last traces of lithic phases of "Paquevira"-roughened stones, "Passasunga" -large objects industry, and "subtradiçao de Bom Jardim". Also present are polished stones, beads (necklaces, earrings, nose-rings, and various jewelry) and microliths.

MC-1029. Chã do Caboclo, Stratum A4

$2800 \pm 95$

Sq 16, level 30 to $40 \mathrm{~cm}$. Comment (AFGL): 1st appearance of lithic material characteristic of "tradiçao ceramica da Pedra do Caboclo".

MC-1084. Chã do Caboclo, Stratum A5

$3650 \pm 115$

Sq 51 , level 40 to $50 \mathrm{~cm}$.

MC-1013. Chã do Caboclo, Stratum A5

$1700 \mathrm{BC}$

Test pit, level 20 to $30 \mathrm{~cm}$.

MC-1044. Chã do Caboclo, Stratum A5

Sq 19, level 20 to $30 \mathrm{~cm}$.

$4460 \pm 100$

2510 BC

$4590 \pm 100$

2640 BC

MC-1043. Chã do Caboclo, Stratum A5

$5540 \pm 100$

Sq 12, level 20 to $30 \mathrm{~cm}$.

3590 BC

MC-1081. Chã do Caboclo, Stratum A5

$5600 \pm 1300$

Sq 53 , level 0 to $10 \mathrm{~cm}$.

3650 вс 


\section{MC-1028. Chã do Caboclo, Stratum A5}

Sq 5 , level 20 to $30 \mathrm{~cm}$. Comment (AFGL): with exception of MC-1084 is too young, all samples come from scattered fire sites of maximum thickness $20 \mathrm{~cm}$. Lithic material of "fase Paquevira, Passasunga and subtradiçao Bom Jardim" are well represented.

\section{MC-1059. Chã do Caboclo, Stratum B}

$6330 \pm 125$

Sq 47 , level 10 to $20 \mathrm{~cm}$.

\section{MC-1061. Chã do Caboclo, Stratum B}

Sq 48 , level 35 to $45 \mathrm{~cm}$.

MC-1045. Chã do Caboclo, Stratum B

Sq 24, level 10 to $20 \mathrm{~cm}$.

MC-1027. Chã do Caboclo, Stratum B

Sq 4, level 30 to $40 \mathrm{~cm}$.

MC-1060. Chã do Caboclo, Stratum B Sq 48 , level $30 \mathrm{~cm}$.

MC-1055. Chã do Caboclo, Stratum B Sq 41, level 30 to $40 \mathrm{~cm}$.

\section{BC}

$6600 \pm 150$

4650 BC

$6900 \pm 135$

4950 BC

$7125 \pm 140$

5175 BC

$7300 \pm 140$

5350 BC

MC-1042. Chã do Caboclo, Stratum B

$7820 \pm 150$

5870 BC

Sq 1, level 30 to $40 \mathrm{~cm}$. Comment (AFGL): only lithic material of "subtradiçao Bom Jardim" was found in stratum, foliaceous arrow heads on one side with convex plan profile, all with grooves; large and short one face triangular points, scrapers, chisels, knife points, microliths.

MC-1056. Chã do Caboclo, Stratum C

$9520 \pm 160$

Sq 42 , level 40 to $50 \mathrm{~cm}$.

MC-1046. Chã do Caboclo, Stratum C

$11,000 \pm 250$ 9050 BC

Sq 31, level 10 to $20 \mathrm{~cm}$. Comment (AFGL): material from this stratum were again characteristic of "subtradiçao Bom Jardim". Most material is still in quartz, but flint, characteristic of older levels, begins to appear.

\section{MC-1087. Chã do Caboclo, Stratum D}

$6820 \pm 190$

Sq 51, level 70 to $100 \mathrm{~cm}$. Comment (AFGL): MC-1087 seems much too young. Sample underlay laterite and was assoc with cutting wastes remaining on quartz gravel. Objects coll in stratum are coarsely finished. 
Comment: Sq 51 did not yield expected results; MC-1082 and -1083, although at same age, statistically are reversed. MC-1084, -1087 are too young. MC-1087 is a regrouping of $1.8 \mathrm{~g}$ charcoal piece, mean surface $1 \mathrm{~mm}^{2}$, sorted out under binocular microscope from $30 \mathrm{~g}$ clay from 2 different levels: 70 to 80 and 90 to $100 \mathrm{~cm}$. During standard pretreatment of sample with $1 \% \mathrm{NaOH},>1 / 2$ was eliminated in fulvic and humic acids.

\section{SEA WATER SAMPLES}

As part of a research project on evolution of pollutants from the sea, daily, weekly, monthly and bi-annual sampling is done at surface and in depth off Monaco. Results of measurements of sea water radiocarbon content of 5 sta are given here. Sampling was done by G Hugues and C Emery Oct 20 to 30, 1975.

Immediately after sampling, 80L are filtered on glass wool and stored in $100 \mathrm{~L}$ barrels in nitrogen atmosphere; $\mathrm{CO}_{2}$ is extracted by acidification at $\mathrm{pH} \mathrm{l}$ with $\mathrm{HCl}$ and bubbling for $6 \mathrm{hr}$ in a close circuit comprising an $0.5 \mathrm{~L}$ bottle of $\mathrm{NaOH} 4 \mathrm{~N} . \delta^{13} \mathrm{C}$ determinations are not made. For all samples, we suppose $\delta^{13} \mathrm{C} \%=1 \pm 1$.

Sta $1 .\left(43^{\circ} 30^{\prime} \mathrm{N}, 8^{\circ} 35^{\prime} \mathrm{E}\right)$

MC-1501. Depth, $50 \mathrm{~m}$

MC-1502. Depth, 200m

MG-1503. Depth, 500m

MC-1504. Depth, 1500m

MC-1505. Depth, 2000m

MC-1506. Depth, 2400m

Sta 2. $\left(42^{\circ} 55^{\prime} N, 8^{\circ} 25^{\prime} \mathrm{E}\right)$

MC-1507. Depth, 50m

MC-1508. Depth, 200m

MC-1509. Depth, $500 \mathrm{~m}$

MC-1510. Depth, 1500m

$$
\begin{aligned}
& \delta^{14} \mathrm{C}=+97 \pm 13 \% \text { 。 } \\
& \Delta=+39 \pm 13 \% \text { 。 } \\
& \delta^{14} \mathrm{C}=+89 \pm 13 \% \\
& \Delta=+32 \pm 13 \% \text { c } \\
& \delta^{14} \mathrm{C}=+57 \pm 11 \% \text { o } \\
& \Delta=+2 \pm 11 \% \text { o } \\
& \delta^{14} \mathrm{C}=+11 \pm 10 \% \text { o } \\
& \Delta=-42 \pm 10 \% \text { 。 } \\
& \delta^{14} \mathrm{C}=+9 \pm 10 \% \text { o } \\
& \Delta=-43 \pm 10 \% \text { o } \\
& \delta^{14} \mathrm{C}=+20 \pm 11 \% \text { o } \\
& \Delta=-33 \pm 11 \% \\
& \delta^{14} \mathrm{C}=+47 \pm 11 \% \\
& \Delta=-8 \pm 12 \% \text { o } \\
& \delta^{14} \mathrm{C}=+25 \pm 11 \% \text { o } \\
& \Delta=-29 \pm 11 \% \text { 。 } \\
& \delta^{14} \mathrm{C}=+24 \text { 느 } 11 \% \text { 。 } \\
& \Delta=-29 \pm 11 \% \text { o } \\
& \delta^{14} \mathrm{C}=-49 \pm 10 \% \\
& \Delta=-98 \pm 10 \% \text { o }
\end{aligned}
$$


MC-1511. Depth, 2000m

MG-1512. Depth, 2500m

Sta 3. $\left(42^{\circ} 22^{\prime} 30^{\prime \prime} N, 7^{\circ} 38^{\prime} E\right)$

MC-1513. Depth, $50 \mathrm{~m}$

MC-1514. Depth, 200m

MC-1515. Depth, 500m

MC-1516. Depth, 1500m

MC-1517. Depth, 2000m

MC-1518. Depth, 2500m

Sta $4 .\left(42^{\circ} 46^{\prime} N, 7^{\circ} \mathrm{E}\right)$

MG-1519. Depth, 50m

MC-1520. Depth, 200m

MG-1521. Depth, 500m

MC-1522. Depth, 1500m

MG-1523. Depth, 2000m

MG-1524. Depth, 2450m

Sta $5 .\left(42^{\circ} 10^{\prime}, 6^{\circ} 40^{\prime} \mathrm{E}\right)$

MC-1525. Depth, 50m

MC-1526. Depth, 200m

MC-1527. Depth, 500m

$$
\begin{aligned}
& \delta^{14} \mathrm{C}=-8 \pm 11 \% \text { o } \\
& \Delta=-59 \pm 11 \% \text { o } \\
& \delta^{14} \mathrm{C}=-18 \pm 10 \% \text { o } \\
& \Delta=-69 \pm 10 \% \text { o }
\end{aligned}
$$

$\delta^{14} \mathrm{C}=+113 \pm 10 \%$

$\Delta=+55 \pm 10 \%$ 。

$\delta^{14} \mathrm{C}=+54 \pm 11 \%$

$\Delta=-1 \pm 12 \%$

$\delta^{14} \mathrm{C}=+56 \pm 13 \%$ o

$\Delta=+1 \pm 13 \%$

$\delta^{14} \mathrm{C}=-3 \pm 10 \%$ o

$\Delta=-55 \pm 10 \%$ 。

$\delta^{14} \mathrm{C}=+17 \pm 11 \%$

$\Delta=-35 \pm 11 \%$

$\delta^{14} \mathrm{C}=+46 \pm 10 \%$ o

$\Delta=-8 \pm 10 \%$

$$
\begin{aligned}
& \delta^{14} \mathrm{C}=+76 \pm 11 \% \circ \\
& \Delta=+20 \pm 12 \% 0 \\
& \delta^{14} \mathrm{C}=+47 \pm 10 \% 0 \\
& \Delta=-7 \pm 10 \% 0 \\
& \delta^{14} \mathrm{C}=-14 \pm 10 \% 0 \\
& \Delta=-66 \pm 10 \% 0 \\
& \delta^{14} \mathrm{C}=-7 \pm 9 \% \\
& \Delta=-59 \pm 9 \% \\
& \delta^{14} \mathrm{C}=+29 \pm 11 \% 0 \\
& \Delta=-24 \pm 11 \% 0 \\
& \delta^{14} \mathrm{C}=+5 \pm 9 \% \\
& \Delta=-47 \pm 9 \% 0 \\
& \delta^{14} \mathrm{C}=+98 \pm 10 \% 0 \\
& \Delta=+41 \pm 10 \% \\
& \delta^{14} \mathrm{C}=+91 \pm 11 \% 0 \\
& \Delta=+35 \pm 12 \% 0 \\
& \delta^{14} \mathrm{C}=+51 \pm 10 \% 0 \\
& \Delta=-3 \pm 11 \%
\end{aligned}
$$


MC-1528. Depth, 1500m

MG-1529. Depth, 2000m

MG-1530. Depth, 2700m

$$
\begin{aligned}
& \delta^{14} \mathrm{C}=+15 \pm 11 \% \circ \\
& \Delta=-37 \pm 11 \% \circ \\
& \delta^{14} \mathrm{C}=0 \pm 10 \% \\
& \Delta=-52 \pm 10 \% \circ \\
& \delta^{14} \mathrm{C}=-2 \pm 11 \% \circ \\
& \Delta=-54 \pm 11 \% \circ
\end{aligned}
$$

IV. ATMOSPHERIC RADIOCARBON ACTIVITY, MONACO

This is a routine control of atmospheric $\mathrm{CO}_{2}$ radiocarbon content. Sample coll from roof of Mus Oceanograph, Monaco $\left(43^{\circ} 13^{\prime} \mathrm{N}, 7^{\circ} 25^{\prime}\right.$ E) May 1975.

\section{MC-1050.}

$$
\delta^{14} \mathrm{C}=+413 \pm 12 \%
$$

\section{REFERENCES}

Bird, J, 1938, Antiquity and migrations of the early inhabitants of Patagonia: Geogr rev, v 28, p 250-275.

Callow, W J, Baker, M J, and Hassall, G I, 1965, National physical laboratory radiocarbon measurements III: Radiocarbon, v 7, p 156-161.

Delibrias, G and Rapaire, J L, 1967, Etude du mouvement propre d'une installation de comptage à bas niveau: Radioactive dating and methods of low level counting: Symposium IAEA, p 603-612.

Delibrias, G, Guillier, M T, and Labeyrie, J, 1972, Gif natural radiocarbon measurements VII: Radiocarbon, v 14, p 280-320.

Laroche, A F G, 1969, Nota brévia sobre um abrigo funerario do Nordeste Brasileiro: Universitas, Univ Fed Bahia, Salvador no. 3-4, May-Dec, p 73-85.

1970, O sitio arqueologico da Pedra do Caboclo: Govt Pernambuco, Sec Edec Cultura, Dept Cultura, Recife, p 78.

1973, Uma pesquisa de salvamento arqueologico na caverna funeraria do Angico PE: Universitas, Univ Fed Bahia, Salvador, no. 14, Jan-April, p 99-120.

1975. Contribucoes para a pré-historia Pernambucana: Govt Pernambuco, Sec Educ Cultura, Recife, p 49.

1976, Os sitios arqueologicos do Angico: Govt Pernambuco: Sec Educ Cultura, Dept Cultura, Recife (in press).

Lobo, P F S, Flexor, J M, Rapaire, J L, and Sieffermann, G, 1974, Essai de détermination du temps de résidence des fractions humiques de deux sols ferrallitiques par l'utilisation du radiocarbone naturel et thermonucléaire: Cah ORSTOM, ser Ped, $\mathrm{v} 12$, no. $1, \mathrm{p} 115-123$.

Ortiz-Troncozo, O, 1971, Investigaciones en Patagonia austral y tierra del fuego: Actas VIth Cong arqueol chilena.

1973, Aspectos arqueologicos de la peninsula de Brunswick: Anales Inst la Patagonia, Punta Arenas, Chile, v 4, p 109-129.

Stuckenrath, $\mathrm{R}$ and Mielke, J E, 1972, Smithsonian Institution radiocarbon measurements VII: Radiocarbon, v. 14, p 401-412.

Stuiver, Minze and Suess, H E, 1966, On the relationship between radiocarbon dates and true samples ages: Radiocarbon, v 8, p 534-540.

Thommeret, J and Rapaire, J L, 1964, Monaco radiocarbon measurements I: Radiocarbon, v 6, p 194-196.

Thommeret, J and Thommeret, Y, 1966, Monaco radiocarbon measurements II: Radiocarbon, v 8, p 286-291.

Turenne, J F, 1975, Modes d'humification et différenciation podzolique dans deux toposéquences guyanaises: Thesis Sci nat OSTROM, Paris, p 191. 